\title{
PIRÓLISE COMO ROTA TECNOLÓGICA PARA APROVEITAMENTO ENERGÉTICO DE BIOMASSAS: PREPARO, PRODUÇÃO E CARACTERIZAÇÃO DE BIO- ÓLEO DE BABAÇU (Orbignya phalerata Martius)
}

Pirolysis as technological route for energetic reclaiming of biomasses: preparation, production and characterization of Babaçu (Orbignya phalerata Martius) bio-oil

La pirólisis como ruta tecnológica para el uso energético de la biomasa: preparación, producción y caracterización del bioaceite de Babaçu (Orbignya phalerata Martius)

\section{Pedro Henrique Fanslau ${ }^{1}$; Gláucia Eliza Gama Vieira ${ }^{1}$; Danilo Gualberto Zavarize²; José} Fernandes de Sousa ${ }^{1}$

${ }^{1}$ Laboratório de Ensaio e Desenvolvimento em Biomassa, Biocombustíveis e Bioenergia, Engenharia Ambiental, Fundação Universidade Federal do Tocantins, Palmas, Brasil.

${ }^{2}$ Unidade de Preparação e Caracterização de Materiais e Biocombustíveis, Doutorado em Ciência dos Materiais, Universidade Federal do Maranhão, Imperatriz, Brasil.

*Correspondência: Laboratório de Ensaio e Desenvolvimento em Biomassa, Biocombustíveis e Bioenergia, Fundação Universidade Federal do Tocantins, Av. NS 15, 109 Norte, Palmas, Tocantins, Brasil. CEP:77.010-090. e-mail pedrohenriquefanslau@gmail.com.

\section{RESUMO}

O Babaçu (Orbignya phalerata Martius) é uma fruta nativa do Brasil, encontrada principalmente nas regiões Norte e Nordeste do país, e também em países que compõem a região Amazônica como a Bolívia, Colômbia e Suriname. A biomassa dessa fruta contém uma diversidade de componentes orgânicos lignocelulósicos, amiláceos e oleaginosos, com grande potencial para obtenção de biocombustíveis. Para tanto, busca-se por rotas tecnológicas de aproveitamento de biomassa como o processo de pirólise, uma técnica promissora capaz de gerar bio-produtos com notável potencial energético. Nesse sentido, o presente estudo aborda o preparo da biomassa e a produção e caracterização do bio-óleo de babaçu, obtido por meio da técnica de pirólise a partir de diferentes partes da fruta. O bio-produto obtido apresentou características como solubilidade, índice de acidez, pH, densidade, viscosidade, corrosividade e poder calorifico superior dentro dos padrões básicos para produção de biocombustíveis como biodiesel, o que indica o potencial de uso dessa matéria-prima.

Palavras-chave: Babaçu; Pirólise; Bio-óleo.

\begin{abstract}
Babassu (Orbignya phalerata Martius) is a native fruit of Brazil, widely found in its North and Northeast regions and also in other countries component of the Amazon region such as Bolivia, Colombia and Suriname. This fruit's biomass contains a diversity of lignocellulosic, starchy and oil-baring organic components with high potential for biofuel production. For that, there has been seeking of technological routes for biomass reclaiming such as pyrolysis, a promising technique able to generate bioproducts with remarkable energetic potential. The present study reports on the preparation of the biomass and the production and characterization of bio-oil from babassu, obtained by pyrolysis of different parts of the fruit. The bioproduct presented characteristics such as solubility, acidity index, $p H$, density, viscosity, corrosivity and higher calorific value in accord to basic standards of biofuels production such as biodiesel, which indicates the potential of this raw material.
\end{abstract}


Keywords: Babassu; Pyrolysis; Bio-oil.

\section{RESUMEN}

El Babaçu (Orbignya phalerata Martius) es una fruta nativa del Brasil, encontrada principalmente en las regiones Norte y Noreste del país, y también en países que componen la región Amazónica como la Bolivia, Colombia y Surinam. La biomasa de esa fruta contén una diversidad de componentes orgánicos lignocelulósicos, almidón y oleaginosos, con alto potencial para obtención de biocombustibles. Por eso, se busca por rutas tecnológicas para el uso energético de la biomasa como el proceso de pirólisis, una técnica promisora capaz de generar bioproductos con notable potencial energético. De esa manera, el presente trabajo se acerca del preparo de la biomasa y la producción y caracterización del bioaceite de babaçu, obtenido por medio de la técnica de pirólisis a partir de las diferentes partes de la fruta. El bioaceite obtenido presentó características como solubilidad, índice de acidez, densidad, viscosidad, corrosividad y valor calorífico superior dentro de los padrones básicos de producción de biocombustibles como el biodiesel, lo que indica el potencial de esa materia prima.

Descriptores: Babaçu; Pirólisis; Bioaceite.

\section{INTRODUÇÃO}

O Brasil tem um grande potencial para o uso de matérias-primas renováveis e é um dos maiores produtores de commodities agrícolas e culturas extrativistas, que geram grandes quantidades de resíduos agroindustriais (VIEIRA et al., 2014). Esses materiais podem ser efetivamente transformados em energia e em outros produtos, por meio de processos integrados que envolvem a conversão da biomassa em uma série de materiais com valor econômico agregado (FOSTER-CARNEIRO et al., 2013).

Devido ao atual cenário de preocupação ambiental, a biomassa se torna um excelente recurso natural de baixo custo e em abundância em todo o mundo (MYTHILI et al., 2013). A produção de energia a partir de biomassa pode ser dividida em duas categorias principais: processos termoquímicos e vias de conversão biológica e, dentre as diversas rotas termoquímicas para produção de energia, está a pirólise (BALAT et al., 2009).

A transformação termoquímica por meio da pirólise ocorre em função de reações químicas em atmosfera inerte, sob diferentes faixas de temperatura, capazes de gerar bio-produtos sólidos (biocarvão), líquidos (óleo e fração aquosa) e gasosos (gases pirolíticos), com mais de $95 \%$ de valorização energética da biomassa aplicada (VIEIRA, 2004).

Estes bio-produtos podem ser aplicados para produção de vapor, eletricidade e novos combustíveis (VIEIRA, 2004). A composição desses bio-produtos depende de inúmeros parâmetros como a matéria-prima de origem e as condições de operação (temperatura, umidade e tempo de residência, etc.) (MORAES et al., 2012).

No processo de pirólise, a formação de biocarvão é favorecida por baixas temperaturas e longo tempo de retenção, enquanto que temperaturas altas e curto tempo de retenção favorecem a formação do bioóleo, e temperaturas muito elevadas e associadas a longo tempo de retenção favorece a formação de gases pirolíticos não-condensáveis como $\mathrm{CO}, \mathrm{CO}_{2}, \mathrm{H}_{2}, \mathrm{CH}_{4}$ (CHANG et al., 2012).

Neste sentido, a pirólise tem sido considerada como uma tecnologia adequada para a reutilização de resíduos agroindustriais para a conversão em energia e, dada a alta demanda por esse recurso e a necessidade de diversificar as fontes da qual é obtido, o babaçu passou então a ser um atrativo na produção de bio-óleo com potencial energético e também outros produtos (TEIXEIRA, 2009; MORAES et al., 2012). 
O Babaçu (Orbygnia phalerata Martius) é uma palmeira nativa da América do Sul, encontrada principalmente nas regiões Norte e Nordeste do Brasil e, em menor medida, na Bolívia, Colômbia e Suriname. O fruto dessa palma é elipsoidal e dividido em epicarpo $(11-13 \%)$, mesocarpo $(20-23 \%)$, endocarpo (5763\%) e grãos/amêndoa (7 - 9\%) (TEIXEIRA, 2009). A abrangência da palmeira de babaçu está entre 13 e 18 milhões de hectares, em 279 municípios situados ao longo de 11 estados, com potencial diverso e inúmeras atividades econômicas desenvolvidas a partir de sua incidência (CARRAZZA et al., 2012).

O babaçu desempenha um importante papel social e econômico para mais de 300.000 famílias extrativistas, as quais obtém sua principal forma de subsistência a partir da quebra manual do coco e extração do fruto (GOMES, 2008).
No processo de extração de óleo, são obtidas duas frações a partir da semente: o óleo e a torta de babaçu. O óleo extraído representa aproximadamente $65 \%$ da massa total da semente e é composto de triglicerídeos e vários outros ácidos graxos livres (ZYLBERSZTAJN et al., 2000).

Nesse sentido, o presente estudo aborda o preparo da biomassa e a produção e caracterização do bio-óleo de babaçu, obtido por meio da técnica de pirólise, a partir de diferentes partes da fruta.

\section{MATERIAIS E MÉTODOS}

O fluxograma ilustrado na Figura 1 apresenta todas as etapas e procedimentos para a coleta, caracterização e análises físico-químicas da biomassa de babaçu e do bio-óleo obtido a partir do processo termoquímico de pirólise.

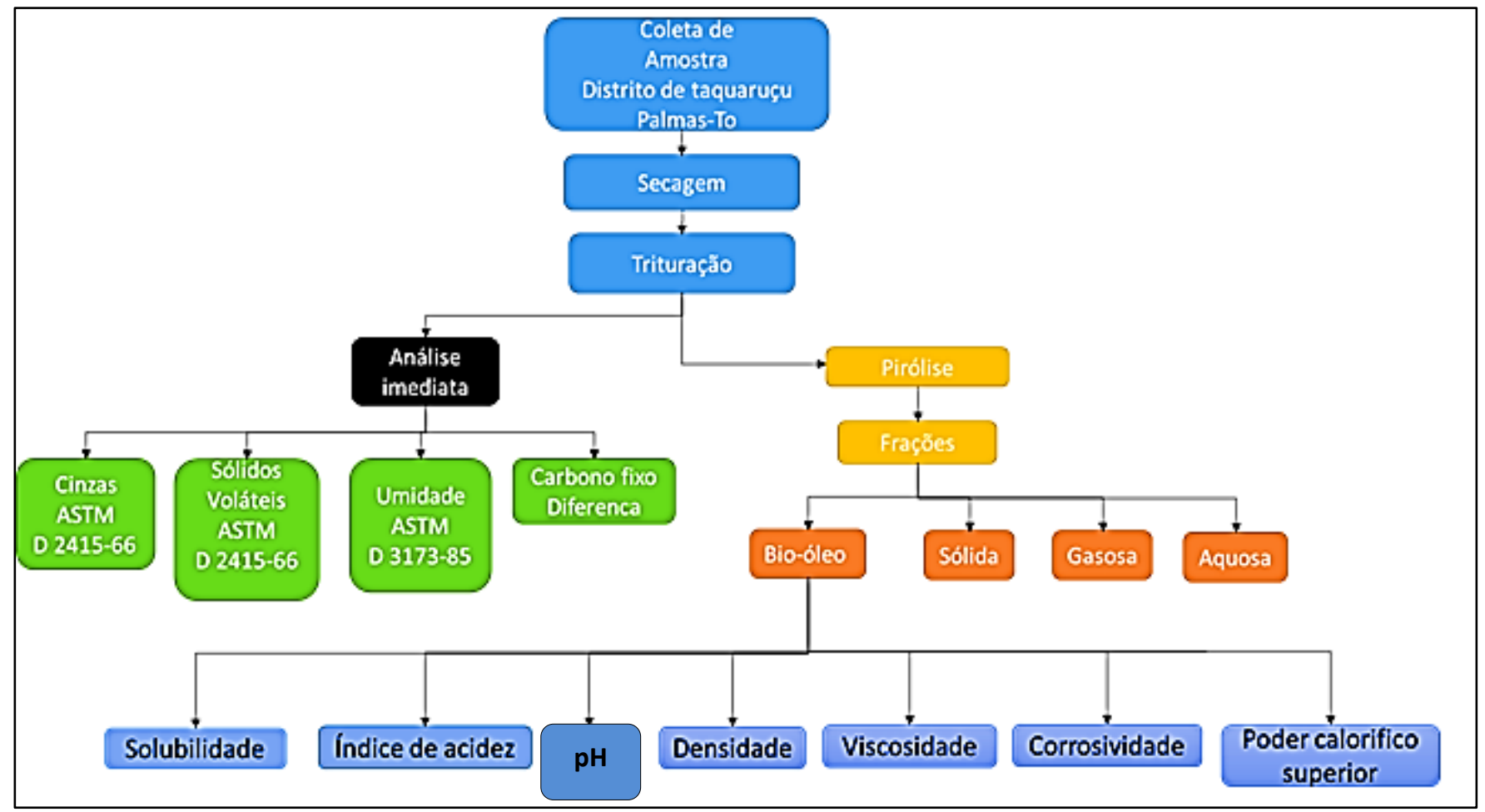

Figura 1. Fluxograma do preparo da biomassa de babaçu e produção e caracterização do bio-óleo (Os autores, 2020)

As amostras foram coletadas manualmente no distrito de Taquaruçú, em Palmas - TO, nas coordenadas UTM 22 L $0813678 \mathrm{~N}$ e $8857122 \mathrm{~S}$

(Figura 2) e levados para o Laboratório de Ensaio e 
Desenvolvimento em Biomassa, Biocombustíveis e Bioenergia - LEDBIO, localizado na Universidade

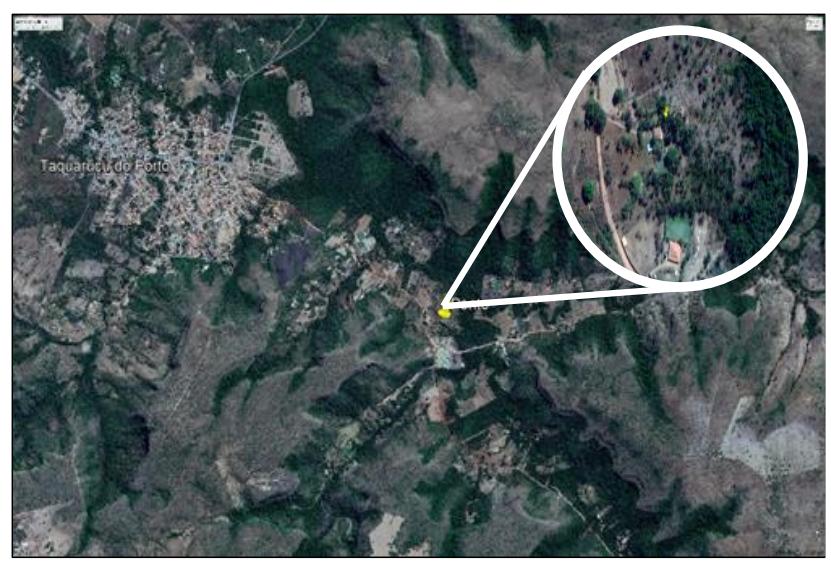

Figura 2. Localização do ponto de coleta no distrito de Taquaruçú em Palmas - TO (Adaptado de Google

Maps, 2020)

\section{Secagem e Preparo da Biomassa}

A secagem da biomassa fracionada em mesocarpo, epicarpo e torta da amêndoa (Figura 3) foi realizada em estufa a $60{ }^{\circ} \mathrm{C}$, de acordo com a metodologia proposta por AOAC (1997).

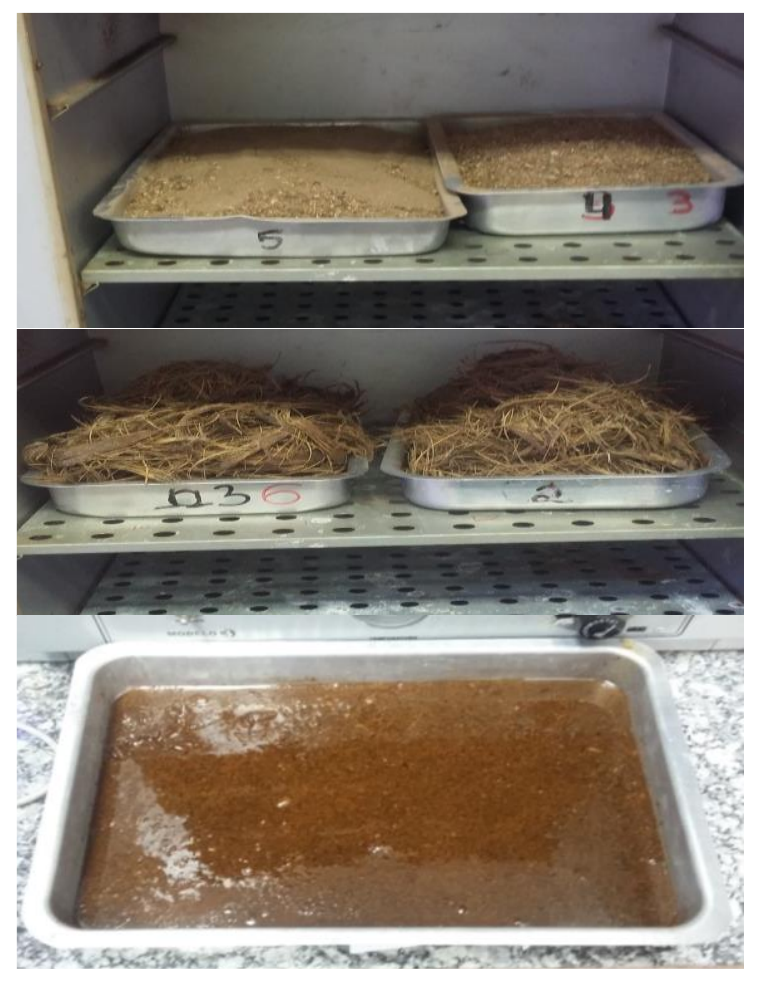

Figura 3. Secagem das partes do fruto em estufa (Os autores, 2020)
Federal do Tocantins - UFT, campus de Palmas, para os processos subsequentes de preparo e caracterização.

Após a secagem, as amostras de mesocarpo, epicarpo e amêndoa de babaçu foram trituradas em um moinho de facas e homogeneizadas em peneiras com abertura de 0,59 mm (ABNT 30/Tyler 28), de acordo com a metodologia utilizada por Colen (2011).

\section{Caracterização Físico-Química}

As amostras moídas e homogeneizadas das partes do babaçu foram submetidas a análises do (i) teor de umidade, (ii) teor de cinzas, (iii) sólidos voláteis e (iv) análise elementar. O teor de carbono fixo foi determinado por diferença.

(i) TEOR DE UMIDADE: determinado de acordo com o método D 3173-85 (ASTM, 1985), no qual uma amostra de $1 \mathrm{~g}$, em triplicata, é colocada em um cadinho de porcelana com peso determinado previamente. Em seguida, as amostras são aquecidas em estufa a $110^{\circ} \mathrm{C}$ durante $1 \mathrm{~h}$ e, em sequência, são colocadas em dessecador por 30 min para resfriamento seguido de pesagem. Esta operação é repetida até peso constante do cadinho, já com a amostra, para determinar a perda de água.

O teor de umidade é determinado de acordo com a Equação 1, em que $P_{o}$ é o peso do cadinho sem a amostra (g), C é o peso da amostra (g) e $P_{1}$ é o peso do cadinho mais a amostra após o aquecimento (g).

Umidade $(\%)=\frac{\left(P_{o}+C\right)-P_{1}}{C} * 100$ (Eq. 1)

(ii) TEOR DE CINZAS: determinado de acordo com o método D 2415-66 (ASTM, 1986), em que uma amostra de $4 \mathrm{~g}$ de cada material, em triplicata, é colocada em cadinhos de porcelana com peso conhecido. Em seguida, se aquece a amostra em forno 
mufla a $900{ }^{\circ} \mathrm{C}$ durante $1 \mathrm{~h}$ e, em sequência, é posta para resfriar em dessecador por $30 \mathrm{~min}$.

O teor de cinzas é o resíduo resultante da parcela da matéria orgânica após carbonização, calculado segundo a Equação 2, em que $\mathrm{P}_{1}$ é o peso do cadinho + cinzas, $\mathrm{P}_{0}$ é o peso do cadinho vazio e $\mathrm{C}$ é o peso da amostra inicial.

Cinzas $(\%)=\frac{\left(P_{1}-P_{0}\right)}{C} * 100$

(iii) SÓLIDOS VOLÁTEIS: os sólidos voláteis (SV) se referem a porção dos sólidos perdida após a ignição ou calcinação da amostra a $550^{\circ} \mathrm{C}$, durante $1 \mathrm{~h}$, para sólidos totais ou dissolvidos voláteis, ou 15 min para sólidos em suspensão voláteis, em forno mufla. A partir do mesmo método D 2415-66 (ASTM, 1986), uma amostra de $1 \mathrm{~g}$ dos materiais foi posta em cadinhos de porcelana com peso conhecido. Em seguida, foi realizado o aquecimento da amostra em uma mufla à uma temperatura de $550^{\circ} \mathrm{C}$ durante $1 \mathrm{~h}$ e, em sequência, colocada para resfriar dessecador por $30 \mathrm{~min}$.

O conteúdo volátil foi calculado segundo a Equação 3, em que que $P_{o}$ é o peso do cadinho sem a amostra (g), C é o peso da amostra (g) e $P_{1}$ é o peso do cadinho mais a amostra após o aquecimento (g).

$S V(\%)=\frac{\left(P_{o}+C\right)-P_{1}}{C} * 100$

(iv) ANÁLISE ELEMENTAR (CNHS): As amostras foram submetidas a análise elementar de Carbono, Nitrogênio, Hidrogênio e Enxofre no equipamento CNHS. A partir dos valores de $\mathrm{C}, \mathrm{N}, \mathrm{He}$ $\mathrm{S}$ foram obtidos as razões atômicas $\mathrm{O} / \mathrm{C}$ e $\mathrm{H} / \mathrm{C}$, e o porcentual de oxigênio determinado por diferença em consideração as cinzas obtidas pelas curvas TGA conforme Equação 4, em que S, H, N e C são os teores de enxofre, hidrogênio, nitrogênio e carbono, respectivamente.

Oxigênio $(\%)=100-(S+H+N+C) \quad$ (Eq. 4)

\section{Pirólise das Amostras}

A pirólise foi realizada em um reator bipartido de leito fixo horizontal, em escala de bancada, constituído por forno modelo FTHI-40 da marca EDG, tubo de alumina, tubo de quartzo, sistema de alimentação de gás inerte, sistema de condensação, sistema de separação de fração líquida e lavadores de gases (Figura 4).

A configuração experimental para todas as amostras foi temperatura de $550{ }^{\circ} \mathrm{C}$, taxa de aquecimento de $10{ }^{\circ} \mathrm{C} \mathrm{min}{ }^{-1} \mathrm{e}$ tempo de retenção de 120 min. Os experimentos foram realizados em triplicata e, ao final do processo, a fração líquida foi coletada a partir do funil de separação de fases. A fração sólida retida dentro da barquinha no interior do tubo foi recuperada e os gases formados durante o processo foram conduzidos por três lavadores em sequência. Todas as fases foram quantificadas para cálculo de rendimento do processo.

\section{Caracterização do Bio-Óleo}

As amostras de bio-óleo obtidas a partir da pirólise de cada parte do babaçu foram caracterizadas quanto a solubilidade, índice de acidez, pH, densidade, viscosidade, corrosividade e poder calorifico superior.

(i) SOLUBILIDADE: O teste foi realizado conforme o método 5520B Partition Gravimetric Method (RICE et al., 2012). 


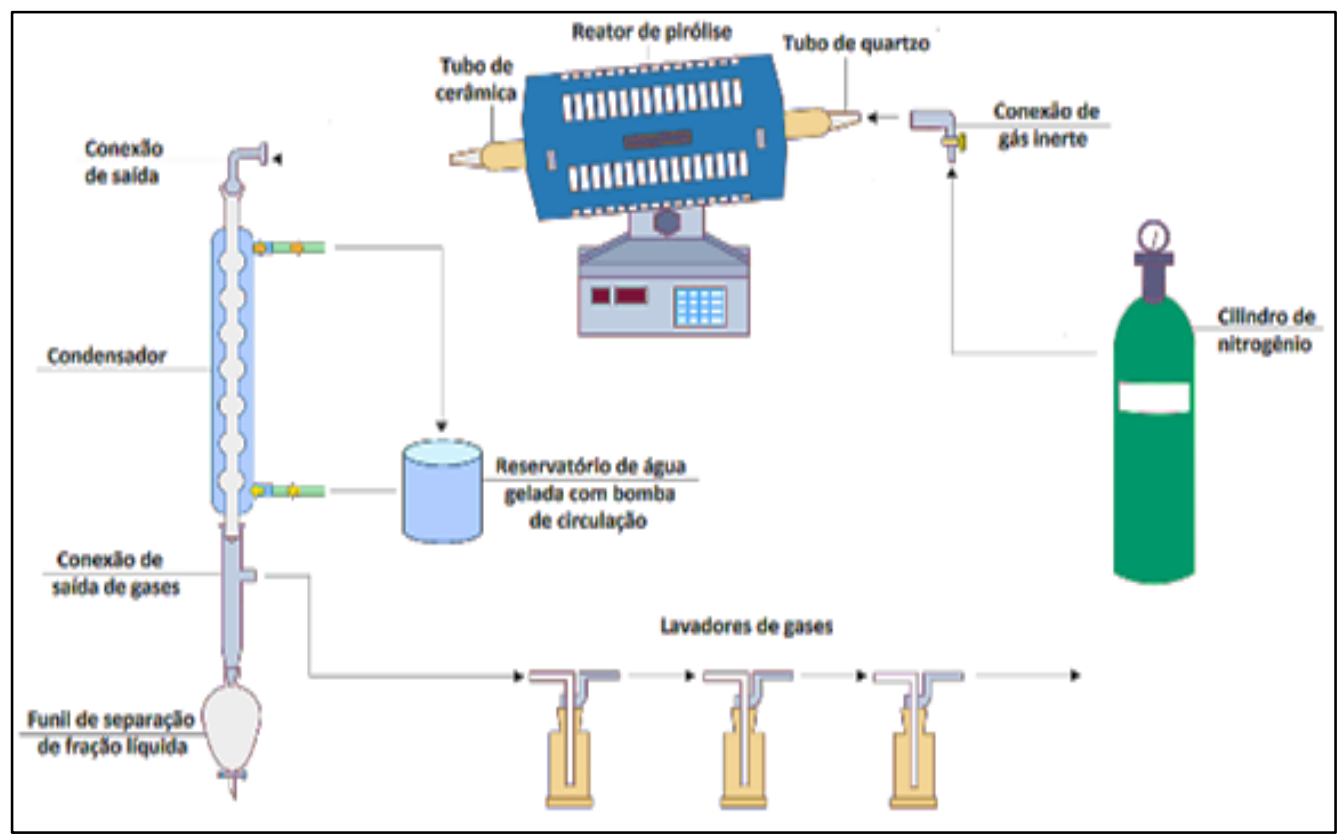

Figura 4. Desenho esquemático da unidade de pirólise em escala laboratorial do LEDBIO - UFT (Fonte: Correia, 2013).

Para a realização do ensaio, colocou-se $2 \mathrm{~mL}$ de amostra em um funil de separação e adicionou-se 30 $\mathrm{mL}$ do solvente. $\mathrm{O}$ funil foi agitado por $2 \mathrm{~min}$ e, em seguida, deixado em repouso para a separação das fases. Após a separação, foi recolhida a fração com a amostra dissolvida no solvente. Esse procedimento foi repetido mais duas vezes.

As frações foram filtradas em um funil contendo $10 \mathrm{~g}$ de sulfato de sódio anidro para um balão previamente pesado. $\mathrm{O}$ solvente foi evaporado utilizando-se um evaporador rotativo (modelo 802, Fisatom). Esse teste foi efetuado para cada uma das amostras - hexano.

A solubilidade foi calculada de acordo com a Equação 5, em que A é a massa do balão com a amostra (g) e B é a massa do balão.

Solubilidade $(g$ de óleo $L)=\frac{(A-B) * 1000}{m L \text { de amostra }}$ (Eq. 5)

(ii) ÍNDICE DE ACIDEZ: o procedimento de titulação para o índice de acidez foi executado de acordo com Zenebon et al. (2008), em triplicata. O método consiste em utilizar solução $0,1 \mathrm{~mol} \mathrm{~L}^{-1}$ de $\mathrm{NaOH}$ para titular o ácido graxo livre na amostra. Em cada amostra são pesados $2 \mathrm{~g}$ de óleo em $255 \mathrm{ml}$ de solução de éter e etanol (2:1) e, após homogeneização da solução, é titulada com indicador fenolftaleína. O índice de acidez é calculado de acordo com a Equação 6 , em que $\mathrm{V}$ é o volume de $\mathrm{NaOH}$ titulado, f é o fator de correção da solução de $\mathrm{NaOH}, 5,61$ é o equivalentegrama de $\mathrm{KOH}$ e $\mathrm{P}$ é o número de gramas da amostra.

Índice de Acidez $=V * f * \frac{5,61}{P}$

(iii) VALOR DE pH: a determinação do $\mathrm{pH}$ foi realizada com auxílio de um pHmetro modelo Q400AS da marca QUIMIS ${ }^{\circledR}$, com eletrodo de vidro $\mathrm{Ag} / \mathrm{AgCl}$ combinado, modelo QA338-ECV e legibilidade de $0,01 \mathrm{pH}$.

(iv) DENSIDADE: a densidade foi calculada por um densímetro portátil da marca DMA 35 (Anton Paar) com uso de $5 \mathrm{~mL}$ de amostra de cada bio-óleo. 
(v) VISCOSIDADE: a viscosidade cinemática foi determinada por meio de banho cinemático modelo EL-BC/219-S. O teste foi realizado fazendo-se o bioóleo escoar, por gravidade, através de um tubo capilar de vidro Cannon-Fenske 250 fixado em garra, calibrado de tal forma que o tempo mínimo de escoamento fosse $200 \mathrm{~s}$, sob temperatura constante de $40{ }^{\circ} \mathrm{C}$, mantida constante no banho cinemático termostático de acordo com o método padrão D 446 (ASTM, 1997). O valor da viscosidade foi determinado conforme a Equação 7, em que $\mathrm{k}$ é a constante cinemática do viscosímetro $(\mathrm{k}=0,25$, para o capilar $), \mathrm{t}$ é o tempo de escoamento da amostra (s) e "e" é a correção da energia cinética para o valor de $\mathrm{T}$.

$V=k *(t-e)$

(iv) CORROSIVIDADE: esse parâmetro foi determinado segundo a metodologia padrão D 130-19 (ASTM, 2019), em que uma placa de cobre foi lixada com auxílio de uma esponja de aço e, depois, inserida em um tubo de ensaio no qual ocorreu a deposição de $10 \mathrm{ml}$ da amostra de bio-óleo, de tal modo que a lamina ficasse parcialmente submersa. $O$ tubo foi acondicionado em um banho maria a temperatura de $100{ }^{\circ} \mathrm{C}$. Após um período de $3 \mathrm{~h}$, observou-se o grau de corrosividade da lâmina de cobre comparando-as a tabela padrão de referência.

(iv) PODER CALORÍFICO SUPERIOR: O PCS das amostras de babaçu e do bio-óleo obtido no processo termoquímico de pirólise foi determinado em bomba calorimétrica da marca IKA, modelo C200. A combustão foi efetuada em excesso de $\mathrm{O}_{2}$ e pressão de 20 a $30 \mathrm{~atm}$.

\section{Análise Estatística dos Dados}

A partir das repetições foram obtidas as médias e desvio-padrão para cada parâmetro analisado. Os valores médios em relação a cada parte da fruta (mesocarpo, epicarpo e amêndoa) para cada parâmetro foram comparados por meio de Teste Tukey ao nível de $5 \%$ de probabilidade, no software R (RCORE TEAM, 2016), para verificar a existência de diferenças estatísticas significativas.

\section{RESULTADOS E DISCUSSÃO}

\section{Caracterização das Amostras de Babaçu}

A caracterização físico-química por meio de análises imediatas é fundamental para o estudo da rota tecnológica de reaproveitamento de resíduos (COLEN, 2011). Neste sentido, elegeu-se para esse estudo as análises de Teor de Umidade, Teor de Voláteis, Teor de Cinzas, Carbono Fixo (por diferença) que, como mencionado previamente, foram conduzidas em triplicata para cada amostra. Os resultados dessas análises experimentais estão apresentados na Tabela 1, juntamente com os valores da análise elementar.

A determinação do teor de umidade é de grande importância na avaliação do consumo energético em processos térmicos, pois quanto maior o teor de umidade no material, maior será o gasto energético envolvido no processo, o que implica em aumento do custo total e, assim, faz-se necessária a secagem prévia da biomassa (COLEN, 2011; ALEXANDRE, 2013).

$\mathrm{O}$ teor médio de umidade nas amostras de mesocarpo, epicarpo e amêndoa do babaçu foi de 5,07, 3,44 e $1,82 \%(\mathrm{~m} / \mathrm{m})$, respectivamente, como visto na Tabela 1, que se apresentaram estatisticamente diferentes. No estudo de Vieira et al. (2014), a torta de amêndoa do babaçu teve um teor de umidade de 3,42\% $(\mathrm{m} / \mathrm{m})$, resultado relativamente próximo ao encontrado no presente estudo, diferente do observado no estudo de Carrazza et al. (2012), no qual foi reportado um teor de umidade de $11,5 \%$ para a mesma parte do fruto. 
Tabela 1. Análises imediatas para as amostras de farinha de mesocarpo, epicarpo e amêndoa do babaçu

\begin{tabular}{cccc}
\hline PARÂMETRO & MESOCARPO & EPICARPO & AMÊNDOA \\
\hline Teor de Umidade (\%) & $5,07 \pm 0,25^{\mathrm{a}}$ & $3,44 \pm 0,37^{\mathrm{b}}$ & $1,82 \pm 0,57^{\mathrm{c}}$ \\
Teor de Sólidos Voláteis (\%) & $85,47 \pm 0,56^{\mathrm{b}}$ & $94,46 \pm 0,97^{\mathrm{a}}$ & $94,28 \pm 0,28^{\mathrm{a}}$ \\
Teor de Cinzas (\%) & $1,55 \pm 0,17^{\mathrm{b}}$ & $1,13 \pm 0,67^{\mathrm{b}}$ & $3,57 \pm 0,21^{\mathrm{a}}$ \\
Carbono Fixo (\%) & $7,90 \pm 0,24^{\mathrm{a}}$ & $0,97 \pm 0,54^{\mathrm{b}}$ & $0,33 \pm 0,14^{\mathrm{c}}$ \\
C (\%) & $39,55 \pm 3,20^{\mathrm{c}}$ & $45,60 \pm 2,4^{\mathrm{b}}$ & $66,28 \pm 3,4^{\mathrm{a}}$ \\
N (\%) & $0,00 \pm 0,00^{\mathrm{a}}$ & $0,00 \pm 0,00^{\mathrm{a}}$ & $0,00 \pm 0,00^{\mathrm{a}}$ \\
H (\%) & $5,62 \pm 1,24^{\mathrm{c}}$ & $6,05 \pm 1,5^{\mathrm{b}}$ & $10,24 \pm 0,21^{\mathrm{a}}$ \\
S (\%) & $0,20 \pm 0,02^{\mathrm{a}}$ & $0,02 \pm 0,008^{\mathrm{c}}$ & $0,11 \pm 0,01^{\mathrm{b}}$ \\
$\mathrm{O}^{1}(\%)$ & $54,63^{\mathrm{a}}$ & $48,33^{\mathrm{b}}$ & $23,25^{\mathrm{c}}$ \\
\hline
\end{tabular}

Por diferença. Médias acompanhadas de letras diferentes, por parte da fruta, indicam haver diferenças estatísticas por meio do Teste Tukey a 5\% de probabilidade.

Em relação ao teor de sólidos voláteis, percebese que não houve diferença estatística entre os valores encontrados para o epicarpo e amêndoa. Dentre os compostos orgânicos que compõe a fração dos sólidos voláteis estão os materiais lignocelulósicos, como a celulose, hemicelulose, lignina, ácidos húmicos e ácidos carboxílicos (PEDROZA, 2011).

Durante a pirólise, os materiais combustíveis, isto é, voláteis, oriundos de biomassas são percebidos principalmente na forma de gases como $\mathrm{CH}_{4}, \mathrm{CO}$ e $\mathrm{H}_{2}$, além de gases não combustíveis, $\mathrm{O}$ e N (VISSOTTO et. al., 2012). Nos estudos de Silva (2009) e Vieira et al. (2014), também para babaçu, reportam-se teores de sólidos voláteis de $87,56 \%$ e $92,32 \% \quad(\mathrm{~m} / \mathrm{m})$, respectivamente, próximos do valor observado nesse estudo. Em comparação, a semente de macaúba, investigada por Nunes (2015), apresentou teor de sólidos voláteis na ordem de $95 \%(\mathrm{~m} / \mathrm{m})$.

Em relação ao teor de cinzas, percebe-se que os valores encontrados estão de acordo com os teores de enxofre (S), principal elemento formador, bem como com os teores de oxigênio $(\mathrm{O})$, obtidos por diferença na análise elementar. Em termos estatísticos, o Teste Tukey ao nível de 5\% de probabilidade apontou não haver diferenças entre os teores obtidos para o mesocarpo e epicarpo. Nos estudos de Vieira et al. (2014) e Nunes (2015) foram observados valores de
$3,93 \%$ e $1,75 \%(\mathrm{~m} / \mathrm{m})$, respectivamente, para esse parâmetro.

Quanto ao carbono fixo, nota-se que o maior valor encontrado foi para o mesocarpo, e que todas as partes que foram avaliadas apresentaram valores estatisticamente diferentes para esse parâmetro. No estudo de Vieira et al. (2014) reporta-se valores de $0,33 \%(\mathrm{~m} / \mathrm{m})$ para carbono fixo, encontrado na torta de amêndoas do babaçu e, no estudo de Nunes (2015), o valor de $0,25 \%(\mathrm{~m} / \mathrm{m})$ para a torta de amêndoa da macaúba. Nesse último estudo, o autor relata que, para o mesocarpo, o valor de carbono fixo foi na ordem de $1,7 \%(\mathrm{~m} / \mathrm{m})$.

A análise elementar indica a composição química de materiais em termos elementares e, segundo Sukiran et al. (2009), pode ser utilizada para determinar parâmetros como requerimentos do ar para combustão, perdas por combustão e, ainda, estimativas dos níveis de emissão de gases. A partir da análise, percebe-se que a biomassa de babaçu é majoritariamente composta por C e O. Em termos estatísticos, todos os teores, com exceção do N, apresentaram-se estatisticamente distintos em relação ao mesocarpo, epicarpo e torta de amêndoas do babaçu. Os valores para teores de $\mathrm{O}$ e $\mathrm{S}$ refletem os teores de cinza encontrados, ao passo que os teores de $\mathrm{C}$ foram inversamente proporcionais aos teores de carbono fixo. 
Análise do Rendimento da Pirólise

A pirolise foi realizada a $550{ }^{\circ} \mathrm{C}$, com taxa de aquecimento de $10{ }^{\circ} \mathrm{C} \min ^{-1}$ e tempo de residência de 120 min. O rendimento do bio-óleo do mesocarpo, epicarpo e torta de amêndoa estão apresentados na Tabela 2.

Tabela 2. Rendimento de bio-óleo na pirólise da biomassa de babaçu

\begin{tabular}{cc}
\hline AMOSTRA & RENDIMENTO $(\boldsymbol{\%})^{\mathbf{1}}$ \\
\hline Mesocarpo & $39,44 \pm 1,2^{\mathrm{c}}$ \\
Epicarpo & $44,02 \pm 1,7^{\mathrm{b}}$ \\
Torta de Amêndoas & $63,36 \pm 2,0^{\mathrm{a}}$ \\
\hline
\end{tabular}

${ }^{1}$ Médias acompanhadas de letras diferentes, por parte da fruta, indicam haver diferenças estatísticas por meio do Teste Tukey a $5 \%$ de probabilidade

Dentre as amostras estudadas nesse trabalho, a torta de amêndoa do babaçu apresentou rendimento superior aos demais, que está atrelado a maior concentração de lipídeos nessa região do fruto (VIEIRA et al., 2014), diferente do epicarpo, que apresenta maior quantidade de lignina, um composto de craqueamento demorado, e também do mesocarpo, que contém carboidratos em maior quantidade, ou seja, uma biomassa de característica amilácea, com cadeias carbônicas longas que tornam o processo quebra demorado e, consequentemente, lento (COLEN, 2011).

Segundo Onorevoli (2016), durante o processo de pirólise de biomassas, as hemiceluloses são degradadas entre as temperaturas de 225 e $325{ }^{\circ} \mathrm{C}$, a celulose entre 305 e $375{ }^{\circ} \mathrm{C}$ e a lignina entre 250 e 500 ${ }^{\circ} \mathrm{C}$. Dessa degradação são produzidos metóxi-fenóis, a partir da lignina, dos quais os monolignóis (guaiacol e siringol) são proeminentes. Da degradação tanto da celulose como da hemicelulose podem ser gerados: levoglucosanos, levoglucosenonas, furfurais, furanos e outras várias moléculas pequenas que compreendem aldeídos e cetonas.

\section{Caracterização do Bio-Óleo}

A análise química e físico-química do bio-óleo utilizado para a produção de biodiesel é importante para atestar a sua qualidade, pois segundo Zavarize (2020), é por meio desses resultados que se estabelece a técnica que será utilizada na sua produção, bem como estabelece se há ou não a necessidade do uso de um prétratamento da matéria-prima. Na Tabela 3 encontramse os dados referentes aos parâmetros de qualidade do bio-óleo obtido do babaçu.

Tabela 3. Caracterização química e físico-química das amostras de bio-óleo de babaçu

\begin{tabular}{cccc}
\hline PARÂMETRO & BIO-ÓLEO MESOCARPO & BIO-ÓLEO EPICARPO & BIO-ÓLEO AMÊNDOA \\
\hline Índice de Acidez (mg & $68,24 \pm 1,25^{\mathrm{a}}$ & $38,78 \pm 2,37^{\mathrm{b}}$ & $1,43 \pm 0,57^{\mathrm{c}}$ \\
de KOH por g) & $28,97 \pm 1,46^{\mathrm{b}}$ & $23,14 \pm 1,97^{\mathrm{b}}$ & $52,98 \pm 1,34^{\mathrm{a}}$ \\
Solubilidade (g de bio- & $2,60 \pm 0,24^{\mathrm{c}}$ & $4,27 \pm 1,02^{\mathrm{b}}$ & $19,81 \pm 1,89^{\mathrm{a}}$ \\
óleo $\left.\mathrm{L}^{-1}\right)$ & $1,21 \pm 0,02^{\mathrm{a}}$ & $1,14 \pm 0,40^{\mathrm{b}}$ & $0,92 \pm 0,03^{\mathrm{c}}$ \\
Viscosidade $\left(\mathrm{mm} \mathrm{s}^{-2}\right)$ & $5,63^{\mathrm{a}}$ & $3,02^{\mathrm{c}}$ & $4,49^{\mathrm{b}}$ \\
Densidade $\left(\mathrm{g} \mathrm{cm}^{-3}\right)$ & $39,64 \pm 0,24^{\mathrm{c}}$ & $50,26 \pm 0,27^{\mathrm{b}}$ & $73,80 \pm 3,20^{\mathrm{a}}$ \\
$\mathrm{pH}$ & $0,00^{\mathrm{a}}$ & $0,00^{\mathrm{a}}$ & $0,00^{\mathrm{a}}$ \\
$\mathrm{C}(\%)$ & $6,65 \pm 1,2^{\mathrm{b}}$ & $6,12 \pm 0,87^{\mathrm{b}}$ & $11,89 \pm 2,84^{\mathrm{a}}$ \\
$\mathrm{N}(\%)$ & $3,54 \pm 1,4^{\mathrm{a}}$ & $0,48 \pm 0,27^{\mathrm{b}}$ & $0,11 \pm 0,02^{\mathrm{b}}$ \\
$\mathrm{H}(\%)$ & $50,17^{\mathrm{a}}$ & $43,14^{\mathrm{b}}$ & $14,20^{\mathrm{c}}$ \\
$\mathrm{S}(\%)$ & $22,92 \pm 2,40^{\mathrm{b}}$ & $23,10 \pm 3,11^{\mathrm{b}}$ & $39,78 \pm 2,5^{\mathrm{a}}$ \\
$\mathrm{O}^{1}(\%)$ & PCS $\left(\mathrm{MJ} \mathrm{kg}^{-1}\right)$ & &
\end{tabular}

Nota: Médias acompanhadas de letras diferentes, por parte da fruta, indicam haver diferenças estatísticas por meio do Teste Tukey a $5 \%$ de probabilidade. 
O índice de acidez é um dos dados mais importantes para avaliação do estado de conservação da matéria-prima, dado que a decomposição de glicerídeos é acelerada pelo calor e pela luz, e a rancidez indicada por esse parâmetro é um sinal consistente da presença de ácidos graxos livres (COLEN, 2011).

A acidez observada para o óleo de amêndoa do babaçu foi 1,43 mg de $\mathrm{KOH}$ por g óleo, já para o mesocarpo e epicarpo a acidez encontrada foi de respectivamente 68,24 e 38,78 mg de $\mathrm{KOH}$ por g óleo, todas as três estatisticamente diferentes. No estudo de produção de biodiesel a partir do óleo de babaçu conduzido por Lima et al. (2007), foi obtido um índice de acidez de 0,224 mg de $\mathrm{KOH}$ por $\mathrm{g}$ de biodiesel, ao passo que, para o bio-óleo, esse valor foi de 1,43 mg de $\mathrm{KOH}$ por g de bio-óleo, igual ao obtido no presente estudo.

Em relação a solubilidade, o bio-óleo de todas as partes do fruto apresentou caráter apolar, parcialmente dissolvido em n-hexano e com formação de fases no funil de decantação. Estatisticamente, a solubilidade do bio-óleo obtido a partir do mesocarpo e do epicarpo não diferiram. O bio-óleo da torta de amêndoa de babaçu apresentou maior concentração de compostos com polaridade similar ao do n-hexano, isto é, uma solubilidade de 52,98 g de óleo. $\mathrm{L}^{-1}$, isto é, entre 43,4 e 56,3\% maior do que aquelas observadas para as outras partes do fruto. Segundo Scheibe (2016), essa diferença entre solubilidades permite a separação dos compostos oleaginosos com solventes de diferentes polaridades, quando feita a análise de composição por cromatografia líquida.

Segundo Colen (2011), quanto a viscosidade cinemática, valores elevados para esse parâmetro sugerem a possibilidade de quedas de pressão nas tubulações que conduzirão esse material, o que pode resultar em vazamentos ou rompimentos e, consequentemente, encarecer os custos de bombeamento.

Quimicamente, esse parâmetro reflete o tamanho das cadeias carbônicas, em quantidade, presentes na amostra. Isto é, quanto maior a viscosidade maiores serão as cadeias carbônicas em número (SUKIRAN et al., 2009). Nas amostras de bioóleo oriundas do mesocarpo, epicarpo e torta de amêndoa do babaçu foram obtidas viscosidades entre 2,60 e 19,81 $\mathrm{mm}^{2} \mathrm{~s}^{-1}$, todas as três estatisticamente diferentes, dentre as quais a torta da amêndoa se destacou pelo maior valor. Segundo Brasil (2008), a viscosidade do biodiesel deve ser entre 3 e $6 \mathrm{~mm}^{2} \mathrm{~s}^{-1}$, ao passo que, nesse trabalho, o bio-óleo com valor mais próximo foi o oriundo do epicarpo apresentou $4,27 \mathrm{~mm}^{2} \mathrm{~s}^{-1}$. Para as outras amostras, deve-se adaptar o processo de conversão em biodiesel a fim de quebrar o maior número possível de cadeia carbônicas longas para que haja maior rendimento de biodiesel. Para efeito comparativo, no estudo de Lima et al. (2007), a viscosidade obtida para o biodiesel de babaçu foi de $4,0 \mathrm{~mm}^{2} \mathrm{~s}^{-1}$.

Quanto a densidade, segundo Pedroza (2011), o processo termoquímico de pirólise tem como objetivo principal obter produtos com densidade energética mais alta e melhores propriedades do que àquelas da biomassa inicial, visto que esses produtos podem ser usados para abastecer energeticamente o próprio processo ou serem comercializados como produtos químicos ou combustíveis. Estatisticamente, as médias de densidade para cada bio-óleo foram todas diferentes. Quimicamente, as diferenças de densidade entre as amostras estão atreladas, segundo Vieira et al. (2014), à composição de hidrocarbonetos e ácidos graxos, ao passo que maiores quantidades de hidrocarbonetos levam a menores densidades e, se for maior a quantidade de ácidos graxos, maior será a densidade. Outro fator de influência, segundo Oasmaa et al. (1997), é a presença de água que, quanto maior 
for, diminuirá proporcionalmente o valor da densidade. Em comparação, no estudo de Bridgwater (2003), obteve-se densidade de $1,02 \mathrm{~g} \mathrm{~cm}^{-3}$ em bioóleo oriundo de materiais lignocelulósicos. No estudo de Lima et al. (2007), o bio-óleo da torta de amêndoa do babaçu teve densidade idêntica à do presente estudo.

Em relação ao pH do bio-óleo de mesocarpo, epicarpo e torta de amêndoa do babaçu, obteve-se os valores 5,63, 3,02 e 4,49, respectivamente, todos de característica ácida e estatisticamente diferentes. Segundo Bridgwater (2003), o pH do bio-óleo oriundo de biomassas lignocelulósicas geralmente varia entre 2 e 4 , atrelado a fatores como características químicas do bio-óleo e as condições experimentais de obtenção. Desta forma, seu uso pode induzir à corrosão de recipientes e tubulações.

Quanto à análise elementar das amostras de bio-óleo, assim como discutido para as amostras de biomassa, os valores obtidos sugerem características essenciais como a estimativa de emissão de gases com potencial poluente (SUKIRAN et al., 2009). Percebese que há predominância dos teores de $\mathrm{C}$ e $\mathrm{O}$, os quais foram estatisticamente diferentes em relação as partes do fruto. Assim como na biomassa, não foram detectados teores de $\mathrm{N}$, e o teor de $\mathrm{S}$ no bio-óleo da torta de amêndoas foi cerca de $97 \%$ menor que aquele encontrado na amostra de bio-óleo do mesocarpo.

$\mathrm{O}$ poder calorífico superior (PCS) indica a quantidade de energia liberada na queima completa de uma amostra do combustível, nesse caso, o bio-óleo (VIEIRA et al., 2014). No presente estudo, o maior valor de PCS obtido dentre as amostras de bio-óleo foi aquele oriundo da torta de amêndoas que, em termos estatísticos, foi o único a se diferenciar dos demais. $\mathrm{Na}$ literatura, o estudo de Nunes (2015) reporta que o bioóleo oriundo da torta de amêndoa da macaúba apresentou PCS de 37,07 $\mathrm{MJ} \mathrm{kg}^{-1}$, em detrimento do PCS do bio-óleo oriundo do mesocarpo, na ordem de
$39,52 \mathrm{MJ} \mathrm{kg}^{-1}$. Bridgwater (2003) sugere que 17,5 MJ $\mathrm{kg}^{-1}$ seja o valor de PCS mais comum entre biomassas lignocelulósicas e, para qualquer valor acima desse, há grande potencial de uso para fins energéticos. Em outros estudos como de Coelho et al. (2005), Soares et al. (2006) e Fiorese et al. (2013), que analisaram o PCS de bio-óleo oriundo da soja, dendê, mamona e girassol, são reportados valores entre 36,7 e 40,1 $\mathrm{MJ} \mathrm{kg}^{-1}$.

Em relação ao parâmetro de corrosividade, os valores encontrados para as amostras de bio-óleo oriundo do mesocarpo, epicarpo e amêndoa de babaçu, ao comparar as lâminas de cobre à escala padrão ASTM D130 foram, respectivamente, $4 b, 4 b$ e 1b, como mostrado na Figura 5.

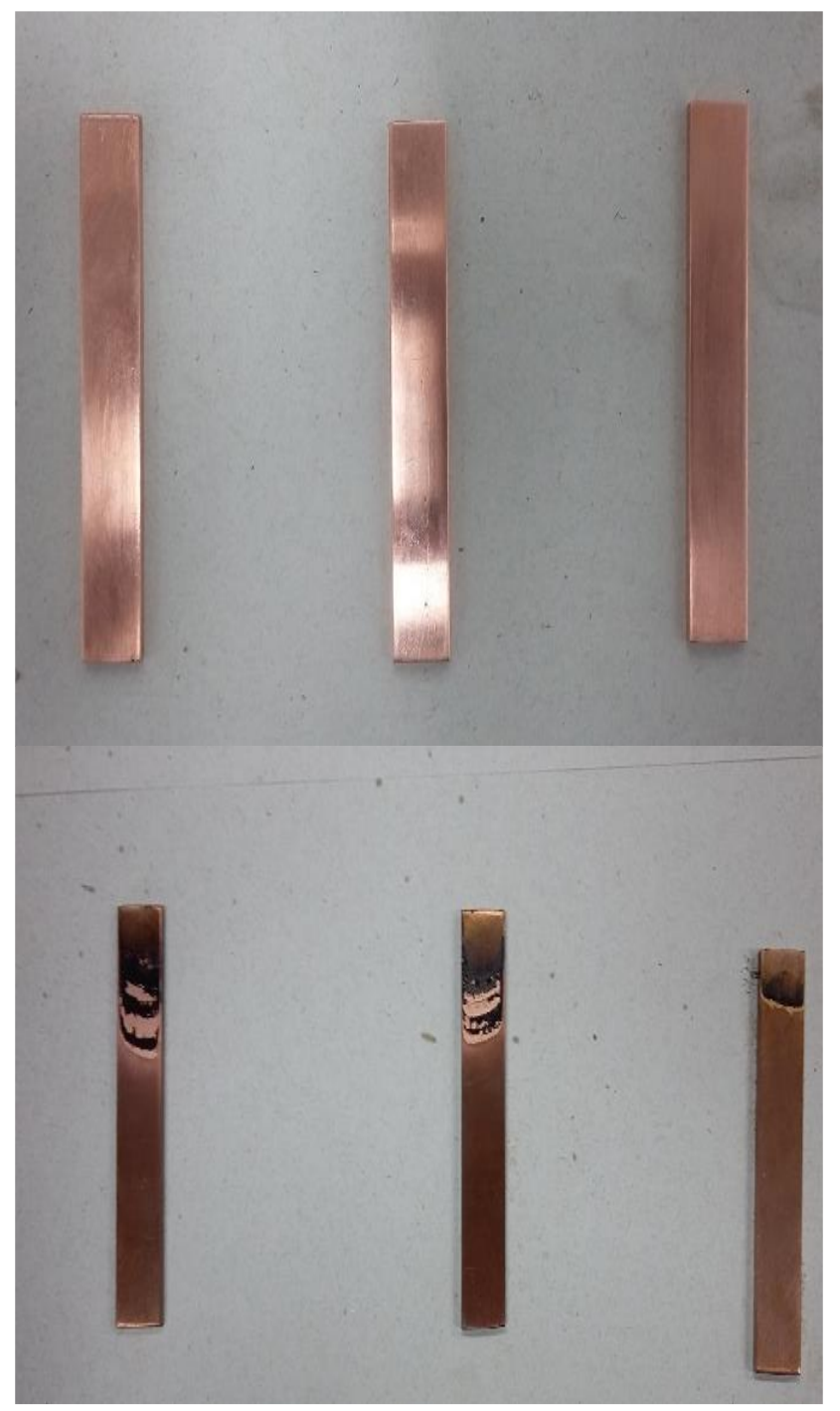

Figura 5. Lâminas de cobre após o ensaio de 
corrosividade com as amostras de bio-óleo de babaçu

(Os autores, 2020)

$\mathrm{O}$ aspecto das lâminas de cobre utilizadas no ensaio com as amostras de bio-óleo do mesocarpo e epicarpo sugere elevada capacidade de corrosão, que pode estar atribuído o elevado índice de acidez e o pH de característica essencialmente ácida dessas amostras. Conforme Dunn et al. (2011), o enxofre é um elemento químico indesejável em combustíveis para motores de combustão, característica marcante nas amostras de bio-óleo obtidas nesse estudo para o mesocarpo e epicarpo de babaçu. Por isso, há grande possibilidade de corrosão das partes metálicas do equipamento como mancais, guias de válvulas, etc. Tal problemática não foi percebida no bio-óleo da torta de amêndoa, o qual apresentou índice de acidez baixo e menor concentração de enxofre dentre as demais amostras, ou seja, apresenta melhores características para produção de biodiesel. Bridgwater et al. (2001) indica que o teor de enxofre em bio-óleo oriundo de biomassas lignocelulósicas é geralmente de 0,2\%, valor relativamente próximo ao encontrado no bioóleo da torta de amêndoa e que se relaciona a uma série de fatores como propriedades do solo, presença de contaminação, disponibilidade de água, trocas químicas na raiz da planta, entre outras.

\section{CONCLUSÃO}

A biomassa do fruto de babaçu apresenta propriedades físico-químicas que corroboram para a produção de bio-óleo com excelente cunho energético, a partir do processo termoquímico de pirólise. As características estudadas indicam conformidade aos padrões necessários para produção de biocombustíveis como biodiesel, principalmente em relação ao potencial calorífico superior, que figura entre os melhores oriundos de biomassas como soja, girassol, dendê, etc. Desta forma, visto a ampla disponibilidade e o apelo socioeconômico, o babaçu pode então ser uma possibilidade na cartilha de biomassas brasileiras aptas à fins energéticos a médio e longo prazo, vista a necessidade de estudos e testes mais detalhados tanto em escala laboratorial como industrial, a fim de obter as melhores condições experimentais de produção.

\section{AGRADECIMENTOS}

Os autores agradecem ao Conselho Nacional de Desenvolvimento Científico e Tecnológico (CNPq) pelo suporte financeiro e aos parceiros de laboratório envolvidos na execução desse estudo.

Todos os autores declararam não haver qualquer potencial conflito de interesses referente a este artigo.

\section{REFERÊNCIAS}

ALEXANDRE, G. P. Avaliação da produção de bioóleo por termocatálise com óxido de cálcio de lodo de esgoto doméstico. $124 \mathrm{f}$ [Dissertação de Mestrado] Programa de Pós-Graduação em Agroenergia Universidade Federal do Tocantins, Palmas, 2013.

AOAC - Association of Official Analytical Chemists. Official Methods of Analysis of AOAC International. 16 Ed. Maryland: AOAC, 1997. 1141 p.

American Society for Testing and Materials (ASTM). D 3173-85: Standard Test of Humidity, 1985.

American Society for Testing and Materials (ASTM). D2415-66: Standard Test Method for Ash Determination. ASTM, 1986.

American Society for Testing and Materials (ASTM). D 446. Standard Specifications and Operating Instructions for Glass Capillary Kinematic Viscometers, 1997.

American Society for Testing and Materials (ASTM). D130-19. Standard Test Method for Corrosiveness to Copper from Petroleum Products by Copper Strip Test, 2019. 
BALAT, M. et al. Main routes for the thermoconversion of biomass into fuels and chemicals. Biomass Bioenergy, v. 32, p. 857 - 864, 2009.

BRASIL. Agência Nacional do Petróleo (ANP). Resolução n. 7 de 19 de março de 2008. Disponível em: <http://www.anp.gov.br>. Acesso em: 25 de agosto de 2018.

BRIDGWATER, A. V. Renewable fuels and chemicals by thermal processing of biomass. Chemical Engineering Journal, v. 91, p. 87 - 102, 2003.

BRIDGWATER, A.V.; CZERNICK, S.; PTRKORZ, 1. An Overview of Fast Pyrolysis. In: Progress in thermochemical biomass conversion. Malden: Blackwell Sciences, 2001.

CARRAZZA, L. R.; ÁVILA, J. C. C.; SILVA, M. L. da. Manual tecnológico de aproveitamento integral do fruto e da folha do babaçu (Attalea spp.). $2 \mathrm{Ed}$. Brasília: Instituto Sociedade, População e Natureza, 68 p., 2012.

CHANG, C.-C.; WU, S.-R.; LIN, C.-C.; WAN, H.-P.; LEE, H.-T. Fast pyrolysis of biomass in pyrolysis gas: fractionation of pyrolysis vapors using a spray of biooil. Energy \& Fuels, v. 26, n. 5, p. 2962-2967, 2012.

COELHO, S. T.; DA SILVA, O. C.; MARIA, S. VELÁZQUEZ, S. G. Uso de óleo de palma "in natura" como combustível em comunidades isoladas da Amazônia. In: III Workshop Brasil-Japão em energia, meio ambiente e desenvolvimento sustentável, 2005, Campinas. Anais... Campinas: 2005. p. 1 - 10.

COLEN, A. G. N. Caracterização físico-química e química do lodo de esgoto para aplicação como fonte de energia em processo de pirólise. $159 \mathrm{f}$. [Dissertação de Mestrado] Programa de PósGraduação em Agroenergia - Universidade Federal do Tocantins, Palmas, 2011.

DUNN, R. O. Fuel properties of biodiesel/ultra-low sulfur diesel (ULSD) blends. Journal of the American Oil Chemists' Society, v. 88, n. 12, p. 1977-1987.

FIORESE, D. A.; FERREIRA, F. M.; SOUZA, S. N. $M$. de. Eficiência energética de um grupo gerador monocilíndrico operando com óleo diesel e biodiesel metílico. Enciclopédia Biosfera, v. 9, n. 17, p. 1241 1251, 2013.

FOSTER-CARNEIRO, T.; BERNI, M. D.; DORILEO, I. L.; ROSTAGNO, M. A. Biorefinery study of availability of agriculture residues and wastes for integrated biorefineries. Resources, Conservation and Recycling, v. 77, p. $78-88,2013$.

GOMES, M. S. S. O. Potencial tecnológico da farinha da amêndoa do coco babaçu (Orbignya sp.) e sua secagem convectiva em leito fixo. Disponível em: https://encurtador.com.br/fvwN7. Acesso em: 26 out. 2017. João Pessoa: UFPB, 2008.

ZENEBON, O.; PASCUET, N. S.; TIGLEA, P. Métodos físico-químicos para análise de alimentos. $4^{\mathrm{a}}$ Ed. (1 ${ }^{\mathrm{a}}$ Versão Digital). São Paulo: Instituto Adolfo Lutz, 2008.

LIMA J. R. O.; SILVA R. B.; SILVA C. M. Biodiesel de babaçu (Orgignya sp.) obtido por via etanólica. Química Nova, v. 30, p. 600-608, 2007.

MORAES, M. S. A.; GEORGES, F.; ALMEIDA, S. R.; DAMASCENO, F. C.; MACIEL, G. P. S.; ZINI, C. A.; JACQUES, R. A.; CAMARÃO, E. B. Analysis of products from pyrolysis of Brazilian sugar cane straw. Fuel Processing Technology. v. 101, p. 35-43, 2012.

MYTHILI, R.; VENKATACHALAM, P.; SUBRAMANIAN, P.; UMA, D. Characterization of bioresidues for biooil production through pyrolysis. Bioresource technology, v. 138, p. 71-78, 2013.

NUNES, A. P. Aproveitamento Sustentável da Macaúba para produção de Biocombustível. $79 \mathrm{f}$. [Dissertação de Mestrado] Programa de PósGraduação em Agroenergia - Universidade Federal do Tocantins, 2015.

OASMAA, A.; LEPPÄMÄKI, E.; KOPONEN, P.; LEVANDER, J.; TAPOLA, E. Physical characterization of biomass-based pyrolysis liquids, Application of standard fuel oil analyses. VTT Publications, v. 306, 87 p. Technical Research Centre of Finland, ESPOO, 1997.

ONOREVOLI, B. Aproveitamento da torta residual obtida como subproduto da extração de óleo das sementes de tabaco energético através da pirólise: caracterização do bio-óleo e biochar. 144 f. [Tese de Doutorado] Programa de Pós-Graduação em Ciência dos Materiais - Universidade Federal do Rio Grande do Sul, Porto Alegre, 2016.

PEDROZA, M. M. Bio-óleo e Biogás da degradação termoquímica de lodo de esgoto doméstico em cilindro rotativo. 192 f. [Tese de Doutorado] Programa de Pós-Graduação em Engenharia Química, Universidade Federal do Rio Grande do Norte, Natal, 2011. 
RCORE TEAM (2016) R: A Language and Environment for Statistical Computing. R Foundation for Statistical Computing, Vienna, Austria. https://www.R-project.org/.

RICE, E. W.; BAIRD, R. B.; EATON, A. D.; CLESCERI, L. S. Standard Methods for the Examination of Water and Wastewater. 22a ed. Washington/EUA: American Public Health Association (APHA), American Water Works Association (AWWA), and Water Environment Federation (WEF), 2012.

SCHEIBE, A. S.; Caracterização de óleos resultantes da pirólise do lodo têxtil. $282 \mathrm{f}$. [Tese de Doutorado] Universidade Federal de Santa Catarina, Centro Tecnológico, Programa de Pós-Graduação em Engenharia Química, Florianópolis, 2016.

SILVA, R. F. da. Avaliação nutricional da torta de babaçu e sua utilização em dietas para frangos de corte label Rouge. 83 f. [Tese de Doutorado] Curso de Ciência Animal, Universidade Federal de Goiás, Goiânia, 2009.

SOARES, B. F.; TAHAN, C. M. V.; PELEFRINI, M. A.; POLIZEL, L. H. Proposição de uso de óleo vegetal in natura e biodiesel de mamona em termelétricas. In: ENC. ENERG. MEIO RURAL, 6, 2006, São Paulo. Anais... São Paulo: Scielo, 2006. v. 6, p. 1 - 10.

SUKIRAN, M. A.; CHIN, C. M.; BAKAR, N. K. A. Bio-oils from pyrolysis of oil palm empty fruit bunches. Am. J. Applied Sci., v. 6, n. 5, p. 869 - 875, 2009.

TEIXEIRA, M. A. Babassu - A new approach for an ancient Brazilian biomass. Part 1: Pyrolysis systems. Energy Conversion and Management, v. 50, p. 3147 $-3157,2009$.

VIEIRA, G. E. G.; PICKLER, A.; GALLO, L. F. P.; TEIXEIRA, L. F.; COLEN, A. G. N.; CAMPOS, C. E. A.; AGUIAR JR, G. L. de; SILVA, R. C. da. Aplicação de processo termoquímico como aproveitamento de resíduo agroindustrial de babaçu. Anais... XX Congresso Brasileiro de Engenharia Química - COBEQ, v. 1, n. 2. São Paulo: Blucher, 2014.

VIEIRA, G. E. G. Fontes Alternativas de Energia Processo de aperfeiçoamento de conversão térmica. 186 f. [Tese de Doutorado] Programa de PósGraduação em Química Orgânica, Universidade Federal Fluminense, Instituto de Química, 2004.

VISSOTTO, J. P.; SANCHEZ, C.; SANCHEZ, E. Caracterização de pinus, eucalyptus, casca de eucalyptus e resíduos florestais e de destoca para fins energéticos. In: VII congresso nacional de engenharia mecânica. São Luís. Anais... São Luís: ABCM, v. 7, p. $1-10,2012$.

ZAVARIZE, D. G. Síntese de catalisador ácido heterogêneo em base carbônica obtida de resíduos de sementes de açaí (Euterpe oleracea Mart.) para produção de biodiesel com de óleo de fritura. $75 \mathrm{f}$. [Dissertação de Mestrado]. Programa de PósGraduação em Agricultura e Ambiente - PPGAA, Universidade Estadual do Maranhão - UEMA.

ZYLBERSZTAJN, D. (Coord.). Reorganização do agronegócio do babaçu no Estado do Maranhão. 108 p. São Paulo: Fundação Instituto de Administração, 2000. 